\title{
The Effect of Asymmetric Mechanical and Thermal Loading on Membrane Wrinkling
} Joseph R. Blandino*

James Madison University

\author{
John D. Johnston ${ }^{* *}$ \\ NASA Goddard Spaceflight Center \\ Jonathan J. Miles ${ }^{* * *}$ \\ James Madison University \\ Urmil K. Dharamsi ${ }^{* * * *}$ \\ James Madison University
}

\begin{abstract}
Large, tensioned membranes are being considered for future gossamer spacecraft systems. Examples include sunshields, solar sails, and membrane optics. In many cases a relatively flat membrane with minimal wrinkling is desired. Developing methods to predict and measure membrane wrinkling is important to the future development of gossamer spacecraft. Numerical and experimental data are presented for a $0.5 \mathrm{~m}$ square, tensioned membrane. The membrane is subjected to symmetric and asymmetric mechanical loading. Data are also presented for a symmetrically loaded membrane subjected to spot heating in the center. The numerical model shows good agreement with the experiment for wrinkle angle data. There is also reasonable agreement for the wrinkled area for both isothermal and elevated temperature tests.
\end{abstract}

\section{Introduction}

Membrane structures typically exhibit nonlinear behavior when loaded. Since a membrane structure cannot support compressive stresses, local buckling or wrinkling often occurs. At ambient temperature the wrinkle pattern is dependent upon both the membrane and loading geometry. At elevated temperature the wrinkle pattern may also be a function of the material properties and the residual stress state of the membrane. The coefficient of thermal expansion will cause the material to expand, while heating may relieve any residual stress from the membrane that was created during manufacturing. Quantifying the wrinkling behavior of a membrane poses many challenges, both experimentally and analytically. The purpose of this paper is to present both experimental and computational data quantifying the wrinkling behavior of a square membrane subjected to symmetric and asymmetric mechanical loading as well as non-uniform thermal loading.

\footnotetext{
"Assistant Professor, Department of Integrated Science and Technology, Member AIAA.

"Aerospace Engineer, Member AIAA.

***Associate Professor, Department of Integrated Science and Technology.

"***Undergraduate Research Assistant, Department of Integrated Science and Technology.
}

\section{Previous Studies}

Membrane structures such as solar sails and sunshields are near-term application of gossamer spacecraft technology. In both cases a relatively flat, reflective membrane surface is desired. Achieving a flat membrane surface is difficult because tensioned membranes will typically undergo wrinkling. The wrinkling occurs because membranes have negligible bending stiffness and cannot support compressive loads. A comprehensive overview of membrane wrinkling is presented by Jenkins and Leonard. ${ }^{1}$ The compressive loads may be due to either mechanical or thermal loading. There have been several studies that have investigated thermal-mechanical effects on membranes. Chiu et. al. ${ }^{2}$ presented an analytical study of wrinkling of a polymer membrane subjected to combined thermal and mechanical loads. This work was not related to spacecraft, but instead was studying the effect of spot heating on transparency films. The work is relevant to this study because the membrane was tensioned and subjected to localized heating at the center of the membrane. Jenkins et. al. ${ }^{3}$ studied the effect of heating on the wrinkling behavior of a round optical membrane. This investigation presents a detailed analysis as well as data from a preliminary experiment. Blandino et al. ${ }^{4}$ presented experimental data on the wrinkling near the corner of a symmetrically tensioned square membrane. Although data was presented for several isothermal cases, the details of an experimental apparatus capable of elevated temperature testing and preliminary data for a heated membrane were also presented.

This paper builds upon previous work by the authors ${ }^{5}$ quantifying the wrinkling of a square membrane subjected to symmetric isothermal loading. A detailed analysis of the wrinkled region and wrinkle angles will be compared with experimental results for both isothermal asymmetric mechanical loading as well as combined symmetric mechanical and asymmetric thermal loading. 


\section{Experiment Design}

Many of the elements that make up the experimental apparatus have been described previously. ${ }^{4,5}$ This section will provide a brief overview of the test specimen, heating system, instrumentation, and load cases.

\section{Test Specimen}

Two test specimens were used for this study. One for each series of tests. Both were a $0.5 \mathrm{~m}$ (19.68 in) square, $2.54 \times 10^{-2} \mathrm{~mm}\left(0.001\right.$ in) thick Kapton ${ }^{\circledR}$ membranes. The membranes have a $1 \times 10^{-3} \mathrm{~mm}$ (3.94 $\times 10^{-5}$ in) vapor deposited aluminum coating on one side. The test articles were cut from a roll of material. The cut section was chosen to be free of either significant material wrinkles or creases. The corners were reinforced with $0.12 \mathrm{~mm}$ (0.005 in) thick transparency film on both sides. Mechanical loads were applied at discrete points at the corners of the membrane via Kevlar ${ }^{\mathbb{Q}}$ threads. Kevlar ${ }^{\mathbb{B}}$ threads were also used to anchor the corners opposite the loads to the test frame. Each membrane was oriented in the load frame such that the manufacturing direction for the rolled material was along the $\mathrm{x}$-axis. The test specimen mounted in the load frame is shown in Fig. 1(a). Details on the load frame are provided in Refs. 4 and 5.

\section{Mechanical Loading}

Mechanical loads were applied by hanging masses from the ends of the Kevlar ${ }^{\circledast}$ threads. This design provided a constant force at all four of the membrane corners. Once the membrane was loaded, the Kevlar ${ }^{\circledR}$ threads were checked to ensure that they were square with the test frame. Excess thread at the top and right side were pulled taut and securely fastened to the test frame so that the membrane could not slip. Prior to hanging the manufacturer's value for each mass was verified using an electronic balance.

\section{Thermal Loading}

Thermal loads were applied to the membrane using a radiant heater. The radiant heater was a flexible heater attached to a $76.2 \mathrm{~mm}$ diameter brass disk with a Silicone RTV designed for use at temperatures up to $200^{\circ} \mathrm{C}$. The face of the disk facing the membrane was painted black. The heater is designed to operate at voltages up to $115 \mathrm{~V}$ and was attached to a variac so that voltages ranging from 0 to $115 \mathrm{~V}$ could be applied. The heater was located in the center of the front face of the membrane. For the elevated temperature tests this was the uncoated side of the membrane. This was done by mounting the heater on a $3.1 \mathrm{~mm}$ diameter ceramic rod, the top of which was inserted into a hole drilled in the brass disk. The bottom of the rod was inserted into an aluminum mounting that attached to the test frame. A picture of the heater is shown in Fig. 1(b).

\section{Test Series}

Two series of tests were run, one under isothermal conditions and another at elevated temperatures. The isothermal tests were repeated with two different membranes. This allowed direct comparison between wrinkle regions from case to case on a single membrane and comparison of wrinkle regions between membranes. The test sequence began with a $2.45 \mathrm{~N}$ load applied. The loading remained unchanged for approximately 15-30 minutes while measurements were taken of the membrane. At the completion of a test case additional mass was added to the left-side corner and the sequence started again until measurements were recorded for each load case. The actual loads applied to the corners are shown in Table 1. For the elevated temperature tests the membrane was loaded symmetrically with $2.45 \mathrm{~N}$.

\section{Membrane Surface Profile Measurements}

Photogrammetry was used to determine the surface profile of the loaded membrane. This method has been demonstrated on membrane structures by the authors and others. ${ }^{5,6}$ A symmetric pattern of target spots was projected onto the non-aluminized side of the membrane using a high intensity light projector and slide. The surface profile is found by accurately determining the location of the center of each target in each image and triangulating. For membrane 1 two sets of images were taken for each load case. The first set of images had targets projected over entire membrane, while the second had targets projected only in the quadrant of the membrane near the left corner. The second membrane had images taken with targets projected over the entire membrane only. For images taken of the entire membrane the target diameter was approximately $3 \mathrm{~mm}$ with targets approximately $3 \mathrm{~mm}$ on center. For images of just the area of the membrane near the left corner the target diameter was approximately $2 \mathrm{~mm}$ and the targets were approximately $2 \mathrm{~mm}$ on center. The two sets of images provided two levels of detail of the membrane surface profile.

Two images were taken with identical, calibrated, digital cameras mounted approximately $610 \mathrm{~mm}$ from the membrane surface. The baseline between the two cameras was approximately $580 \mathrm{~mm}$. The cameras had a $2048 \times 1536$ pixel resolution. A frame with several targets surrounded the membrane. These $12.5 \mathrm{~mm}$ diameter targets were used to scale the images and orient the axes. The frame with respect to the membrane was shown in Fig. 1(a). The targets on the frame are also used within the program to determine camera rotations and the angle between the camera and the image. 
It is important to note that to a certain extent the accuracy of results obtained using photogrammetry are dependent on the target size and target density. Each target must have a sufficient number of pixels to allow accurate determination of the center, yet the target density must be large enough to have a sufficient number of data points to resolve details in the wrinkle patterns. For imaging the entire membrane with the 3.1 megapixel cameras used for this study the minimum target size is approximately $1.8 \mathrm{~mm}$. The desired target size for highest accuracy is approximately $7 \mathrm{~mm}$. Unfortunately, if $7 \mathrm{~mm}$ diameter targets were used for this project the target density would have been too course to resolve any wrinkle details. The projector and slide format used were not capable of projecting 1.8 $\mathrm{mm}$ targets across the entire membrane. If only a quadrant of the membrane is imaged the minimum target size becomes approximately $1.0 \mathrm{~mm}$ and the ideal target size is approximately $3.5 \mathrm{~mm}$. The method for determining ideal target sizes is given in Ref. 7 . Details such as wrinkle amplitude and wrinkle angle are best determined using images of only a membrane quadrant while wrinkled region can be determined from images of the entire membrane. Another factor affecting the accuracy of the membrane profile is target illumination. As discussed previously accuracy depends on determining the center of a target in two images. Thus it is desirable to have uniform illumination across the membrane surface. Projecting targets, even on the non-aluminized side of the membrane, produced glare and non-uniform contrast across the membrane. Therefore the membrane was coated with a thin layer of a talc-based developer solution. The solution dried white and adhered but did not bond to the membrane surface. The coating provided a non-reflective surface to project targets onto the membrane.

\section{Membrane Surface Temperature Measurements}

The temperature profile of the heated membrane was acquired using an Agema 870 Thermovision ${ }^{\circledR}$ infrared scanning imager. The imager head uses a TE-cooled $\mathrm{HgCdTe}$ detector and is sensitive in the $8-12 \mu \mathrm{m}$ waveband. The image produced comprises $144 \times 144$ pixels and is interlaced $4: 1$, with each line refreshed $6-$ $1 / 4$ times per second. Image processing was accomplished using a dedicated software package that accepts an estimated averaged target emissivity value and ambient and atmospheric temperatures that are incorporated into the conversion algorithm that converts raw radiosity data into temperatures. We recognize that the aluminum coated membrane surface exhibits a particularly low spectral emittance profile. In order to maximize spatial resolution the imager was placed one meter from the rear of the membrane, the shortest distance that would allow for the entire temperature profile to be in view. A canopy was placed above and below the imager to control stray thermal emissions, a necessary step given the low temperatures and emittance associated with the membrane. A stream of 100 images was integrated to produce a single image with lower noise associated with each pixel. The Agema system does not adjust for emittance spectrally, but rather invokes a single averaged value. Several flat black dots were painted on the membrane and then it was heated under the same conditions as during the test. Given that the black spots would emit nearly like a blackbody, we were able to back out an estimated average emissivity value by comparison of the images collected before and after the spots were applied. This results in a repeatable emissivity value of 0.115 . This was assumed constant across the entire membrane

\section{Experimental Results}

The goal of this research was to quantify the wrinkled region of a square membrane subjected to asymmetric mechanical loading as well as non-uniform thermal loading. This section presents experimental data for the isothermal mechanical loading as well as elevated temperature tests.

\section{Isothermal Mechanical Loading.}

Contour plots of the left corner of membrane 1 for both load cases are presented in Fig. 2(a)-(b). Figure 2(a) shows the wrinkle patterns for membrane 1 subjected to a symmetric $2.45 \mathrm{~N}$ load. Figure 2(b) shows the same membrane subjected to an asymmetric $2.45 \mathrm{~N} \times 7.36 \mathrm{~N}$ load. Note that the load is applied at $X=0, Y=0$ in these plots. In both plots the contour lines show $0.05 \mathrm{~mm}$ changes in surface amplitude. Figure 3 shows some bad pixel data near the $X$-axis but several wrinkles are clearly visible in both Fig. 2. A program was written in LabVIEW that determined the $X$ and $Y$ coordinates of the wrinkle peaks and valleys at various cross sections. The program then used this data to determine the angle of the peaks and valleys with respect to the $\mathrm{X}$-axis. Table 2 provides data on the angles with respect to the $\mathrm{X}$-axis for peaks and valleys for both load cases.

The wrinkle amplitudes from membrane 1 are compared for both load cases in Fig. 3. The wrinkle amplitude for the symmetric case is similar to that reported by the authors in Ref. 5. Figure 3(a) shows a cross section of the membrane plotted from data taken along a line from $X=100 \mathrm{~mm}$ to $Y=100 \mathrm{~mm}$. Figure 3 (b). shows a cross section of the membrane plotted from data taken along a line from $X=150 \mathrm{~mm}$ to $Y=150 \mathrm{~mm}$. Diagonal cross sections are chosen because they are approximately perpendicular to the wrinkling. Figure 3 shows that as the loading ratio is increased there is a slight decrease in the wrinkle amplitude and the wrinkle wavelength. 
Figures 4-5 compares the two different membranes using photogrammetry results for the entire membrane. Figure 4 compares the symmetrically loaded membranes, while Fig. 5 compares the two membranes subjected to asymmetric loading. Comparisons can also be made between the two figures for similar membranes subjected to different loads. It is important to note, for the reasons discussed in the experiment design section, that these figures will not show all the wrinkles present in the membrane surface profile. What these figures will show is relatively large amplitude, large wavelength wrinkles. They are useful for making general statements about the wrinkled region of the membrane. Figure 4 shows that the wrinkling is primarily confined to a region within $200 \mathrm{~mm}$ of the corners. Wrinkling is apparent on each membrane near all four corners. Figure 5 shows that as the load ratio increases from $1: 1$ to $1: 3$ the wrinkled region is larger near the corners of increased loading (the corners $X=0, Y=0$ and $X=500, Y=500$ ). The wrinkled region near these corners is larger than that for the symmetrically loaded membranes. The wrinkled region occurs within $250 \mathrm{~mm}$ of the corners.

\section{Elevated Temperature Tests.}

To study the effect of thermal loading on membrane wrinkling membrane 2 was loaded symmetrically with $2.45 \mathrm{~N}$ loads and a spot heater was used to apply a nonuniform thermal load to the center of the membrane. The temperature distribution near the center of the membrane is shown in Fig. 6. The figure shows that the temperature drops off rapidly away from the heated area. The maximum temperature was approximately $355 \mathrm{~K}$. The temperature near the edges of the membrane was approximately 293 K. Figure 7 shows a comparison between the surface profiles for the heated and unheated membranes. Contour lines are shown every $0.05 \mathrm{~mm}$. The most distinctive feature, shown in Fig. 7(b) is the depression in the membrane in the heated area. This depression is 4-5 times larger in amplitude than the largest wrinkles near the edges. Slight changes are also visible in the wrinkled region between the heated and unheated membranes. The wrinkled region, particularly near the left corner, appears less distinct on the heated membrane.

\section{Analysis of Experiments}

An analysis was performed to predict the membrane wrinkling behavior studied in the laboratory experiments. The following sections discuss the technique used to analyze the wrinkled membrane, details of the finite element model, and predictions for the membrane structural response under symmetric mechanical, asymmetric mechanical, and combined symmetric mechanical and thermal loading. Analysis Technique
Nonlinear analysis techniques are required in order to accurately account for geometric nonlinearities, load stiffening, and wrinkling effects in the analysis of membrane structures. The present analysis is performed using the commercially available finite element analysis program ABAQUS. ${ }^{8}$ A nonlinear static analysis with membrane wrinkling effects accounted for through the use of a user defined material (UMAT) subroutine is used to predict the structural response of the membrane. The formulation utilized here was developed by Adler ${ }^{9}$ based on work by Miller and Hedgepeth. ${ }^{10}$ In this approach, membrane element material properties are iteratively modified during the analysis to account for the effects of wrinkling and slackness. The analysis procedure is as follows. First, a finite element model of the structure is generated using membrane elements that are assigned the wrinkling material model. Next, a nonlinear static analysis of the structure is performed during which the state of each element (taut, slack, or wrinkled) is determined. A combined stress-strain criteria is used in the UMAT to determine the element states. Following the elements state determination, the stiffness matrix of each element is updated as follows. If the element state is taut, the stiffness matrix is unaltered. If the element state is slack, the stiffness matrix is set equal to zero. If the element state is wrinkled, the stiffness matrix is modified according to Stein-Hedgepeth wrinkling theory. ${ }^{11}$ Stein-Hedgepeth wrinkling theory predicts that the stress state in a wrinkled region of a membrane is uni-axial (positive major principal stress and zero minor principal stress) and wrinkles form in straight lines along the direction of the major principal stresses (load transfer in the wrinkled region is along these lines). Note that the theory predicts average strains and displacements (not individual wrinkle details such as amplitude and wavelength) in the wrinkled region. Further details regarding the UMAT developed by Adler can be found in Ref. 12. This analysis procedure was previously used by the authors to predict the wrinkling behavior of a square membrane subject to corner loads. ${ }^{5}$

In the current paper, this work will be extended to consider combined mechanical and thermal loading. A sequentially coupled thermal stress analysis is performed to predict the response of the membrane to combined mechanical and thermal loading. A steadystate heat transfer is first performed using a heat transfer model to predict the temperature distribution in the membrane. Next, a nonlinear static structural analysis is performed in two steps. In the first step the mechanical preloads are applied to the structure. In the second step the thermal loads are applied by prescribing the temperature results from the heat transfer analysis. 


\section{Finite Element Model}

The finite element model of the laboratory experiment includes representations of the membrane, corner reinforcements, and Kevlar threads. The membrane is modeled using ABAQUS M3D4 and M3D3 membrane elements which are assigned the wrinkling material model. The corners are modeled with ABAQUS S3R shell elements, and the Kevlar threads are represented using ABAQUS B31 beam elements. The boundary conditions are applied at the ends of the Kevlar threads attached to the test fixture. The ends of the top and left threads are constrained in all three translational degrees of freedom, while the right and bottom threads are constrained in the two translational degrees of freedom normal to the loading direction. The mechanical loads are applied as concentrated loads at the ends of the right and bottom Kevlar threads. Thermal loads are applied as predefined temperatures at the grid points. The initial temperature of all grids is taken to be $293 \mathrm{~K}$

\section{Analytical Predictions}

The structural response of the membrane was analyzed for three cases: (1) symmetric mechanical loading at room temperature, (2) asymmetrical mechanical preloading at room temperature, and (3) combined symmetric mechanical and thermal loading. Table 3 summarizes the maximum stress and the stress at the center of the membrane for each of the load cases. Figure 8 presents plots of the temperature distributions in the membrane for each of the load cases. Figures 9. 11 present plots of analytical predictions for the major principal stresses, minor principal stresses and wrinkled/slack regions, respectively for each of the load cases. These wrinkle/slack region plots were generated by outputting the element integration point states from the final step of the analysis.

In case 1 the membrane is subjected to symmetric mechanical loading of $2.45 \mathrm{~N}$. The membrane has a uniform temperature of $293 \mathrm{~K}$, Fig. 8(a), i.e. the membrane is at room temperature. The plot of the major principal stresses, Fig. 9(a), shows that there are stress concentrations at the corners of the membrane where the mechanical loads are applied. The minor principal stress plot, Fig. 10(a), predicts that there is a region of near-zero minor principal stress around the corners and outside edges of the membrane. The wrinkle region, Fig. 11(a), is seen to extend from the corners around the edges of the membrane corresponding to the region of zero minor principal stresses. In case 2 the membrane is subjected to asymmetric mechanical loading of $2.45 \mathrm{~N}$ in the vertical direction and $7.35 \mathrm{~N}$ in the vertical direction. As with load case 1 , the membrane has a uniform temperature of $293 \mathrm{~K}$, Fig. 8(b). The plot of the major principal stresses, Fig. 9(b), shows that the stresses in the horizontal direction have increased corresponding to the increase in horizontal preload. The minor principal stress plot, Fig. 10(b), predicts that the region of near-zero minor principal stresses expands. The wrinkle region, Fig. 11(b), is seen to expand in the left and right corners and to now include a band across the center of the membrane. In case 3 the membrane is subjected to symmetric mechanical loading of $2.45 \mathrm{~N}$. The membrane has a non-uniform temperature distribution, Fig. 8(c). This temperature distribution corresponds to the spot heating laboratory experiment that resulted in a 'bulls-eye' temperature distribution with a maximum temperature of $355 \mathrm{~K}$ at the center of the membrane. The plot of the major principal stresses, Fig. 9(c), shows a significant change in the stress distribution compared to load case 1 . The center of the membrane now has a large region of near-zero major principal stresses. The minor principal stress plot, Fig. 10(c), also shows major changes compared to load case 1. The region of near-zero minor principal stresses now encompasses all of the membrane except for four ovalshaped regions near the four corners. Figure 11(c) shows that there are both wrinkled and slack regions present in the membrane. The slack region is roughly square in shape and is located in the center of the
membrane.

\section{Discussion}

Predictions from the finite element analysis were compared with results from the laboratory experiments. The analysis is useful in predicting wrinkle regions and wrinkle direction, however it does not predict wrinkle details such as amplitude and wavelength. Figure 12 compares the wrinkle angle data from Table 2 with the predicted values from the analysis. The comparison is made between the predicted major principal stress directions and the measured wrinkle angles (for both the troughs and crests of the wrinkles). Both the angular measurements are taken from the $\mathrm{x}$-axis as defined in Fig. 1. Recall that Stein-Hedgepeth theory predicts that wrinkles form in straight lines along the direction of the major principal stresses, thus the measured wrinkle angles are expected to fall along the predicted major principal stress angles. Figure 12(a) shows a comparison for the symmetrically loaded membrane, while Fig. 12(b) compares the asymmetrically loaded membrane. In both cases there is good agreement between the predicted and measured wrinkle angles. This data also agrees with data presented by the authors in Ref. 4. Comparing Figs. 4 and 11(a) show that the analysis under-predicts the size of the wrinkled area for the symmetrically loaded membrane. The experiments show that the wrinkled region extends approximately $200 \mathrm{~mm}$ from each corner. The analysis shows wrinkling within approximately $125 \mathrm{~mm}$ from each corner. The agreement between experiments and analysis is better for the asymmetric load case. 
Comparing Figs. 5 and 11 (b) for the $2.45 \mathrm{~N} \times 7.36 \mathrm{~N}$ load case shows that the analysis predicts that the wrinkled regions near the top and bottom of the membrane will shrink and the wrinkling will extend across the membrane from the left and right corners. Although the experiments do not show the wrinkling extending across the membrane the data show that the wrinkled regions at the top and bottom of the membrane do shrink in size. The experiments also show that the wrinkled area near the corners extends out to approximately $250 \mathrm{~mm}$. The data presented here and in Ref. 5 verify that the numerical technique is viable for use over a range of isothermal load cases.

The experimental data also show that the analysis technique is useful for predicting combined thermalstructural behavior. The analysis predicted a relatively large slack area near the center of the membrane as was shown in Fig. 11(c). The experimental results shown in Fig. 7(b) show a large amplitude depression within the heated area of the membrane that roughly corresponds to the location of the predicted slack region. Although the results need to be verified and the tests performed in under vacuum conditions to eliminate convection, the comparison between. experiment and analysis is promising. The slack region develops due to overexpansion of the center of the membrane (where the thermally-induced strains are the greatest) compared to the outer edges. Previous studies by the authors have shown that the stresses and wrinkle region predicted for the case of symmetric mechanical loading and a uniform elevated temperature membrane are the same as those predicted for a mechanical load only (i.e. room temperature) analysis. This is due to the "constant force' preloading scheme which is intended to accommodate thermal expansion/contraction of the membrane. Interestingly, the results of this study show that the constant force preloading scheme is less effective in the presence of non-uniform temperature distributions. In general, it is expected that large membrane structures for space applications such as sunshields will not have uniform temperatures, and thus careful attention should be given to these thermalstructural effects.

\section{Summary}

This paper has described a combined experimental and analytical study of membrane wrinkling for the cases of symmetric and asymmetric isothermal mechanical loading and combined symmetric mechanical loading and non-uniform thermal loading. A comparison of experimental results and analytical predictions for the wrinkle directions and wrinkle regions shows fair agreement.

\section{Acknowledgement}

This research has been supported in part by NASA Goddard Space Flight Center Grant NAG5-10701.

\section{References}

1. Jenkins, C.H. and Leonard, J.W., "Nonlinear Dynamic Response of Membranes: State of the Art," Applied Mechanics Reviews, American Society of Mechanical Engineers, Vol. 44, No. 7, July 1991, pp. 319-328.

2. Chiu, H.C., Benson, R.C., Fiscella, M.D., and Burns, S.J., "Mechanical and Thermal Wrinkling of Polymer Membranes". Journal of Applied Mechanics, Vol. 61, March 1994, pp. 67-70.

3. Jenkins, C.H., Fitzgerald, D.M., Liu, X., "Wrinkling of an Inflatable Membrane with Thermo-Elastic Boundary Conditions," $41^{\text {st }}$ AIAA Structures, Structural Dynamics, and Materials Conference, Atlanta, GA, Paper No. AIAA-2000-1727, April 2000.

4. Blandino, J.R., Johnston, J.D., Miles, J.J., Soplop, J.S., "Thin-Film Membrane Wrinkling due to Thermal and Mechanical Loads," $42^{\text {nd }}$ AIAA Structures, Structural Dynamics and Materials Conference, Seattle, WA, Paper No. AIAA-2001-1345, April 2001. 5. Blandino, J.R., Johnston, J.D., and Dharamsi, U.K., "Corner Wrinkling of a Square Membrane due to Symmetric Mechanical Loads," submitted to Journal of Spacecraft and Rockets, July 2001.

6. Pappa, R.S., Giersch, L.R. and Quagliaroli, J.M.,"Photogrammetry of a 5m Inflatable Space Antenna with Consumer Digital Cameras," NASA TM2000-210627. Dec. 2000, pp. 16.

7. Photomodeler User Manual, Version 4.0, EOS Systems, Inc. Vancover, B.C., Canada. 2002.

8. ABAQUS /Standard User's Manual, Version 6.1, Hibbitt, Karlsson, \& Sorensen, Inc., Pawtucket, RI, 2000.

9. Adler, A.L., Mikulas, M.M., and Hedgepeth, J.M., "Static and Dynamic Analysis of Partially Wrinkled Membrane Structures," $41^{\text {st }}$ AIAA Structures, Structural Dynamics, and Materials Conference, Atlanta, GA, Paper No. AIAA-2000-1810, April 2000.

10. Miller, R.K. and Hedgepeth, J.M., "An Algorithm for Finite Element Analysis of Partly Wrinkled Membrane Structures," AIAA Journal, Vol. 20, No. 12, December 1982, pp. 1761-176

11. Stein, M. and Hedgepeth, J.M., "Analysis of Partly Wrinkled Membranes”. NASA TN D-813, 1961.

12. Adler, A.L., "Finite Element Approaches for Static and Dynamic Analysis of Partially Wrinkled Membrane Structures," PhD Thesis, Department of Aerospace Engineering, University of Colorado, Boulder, CO, 2000. 

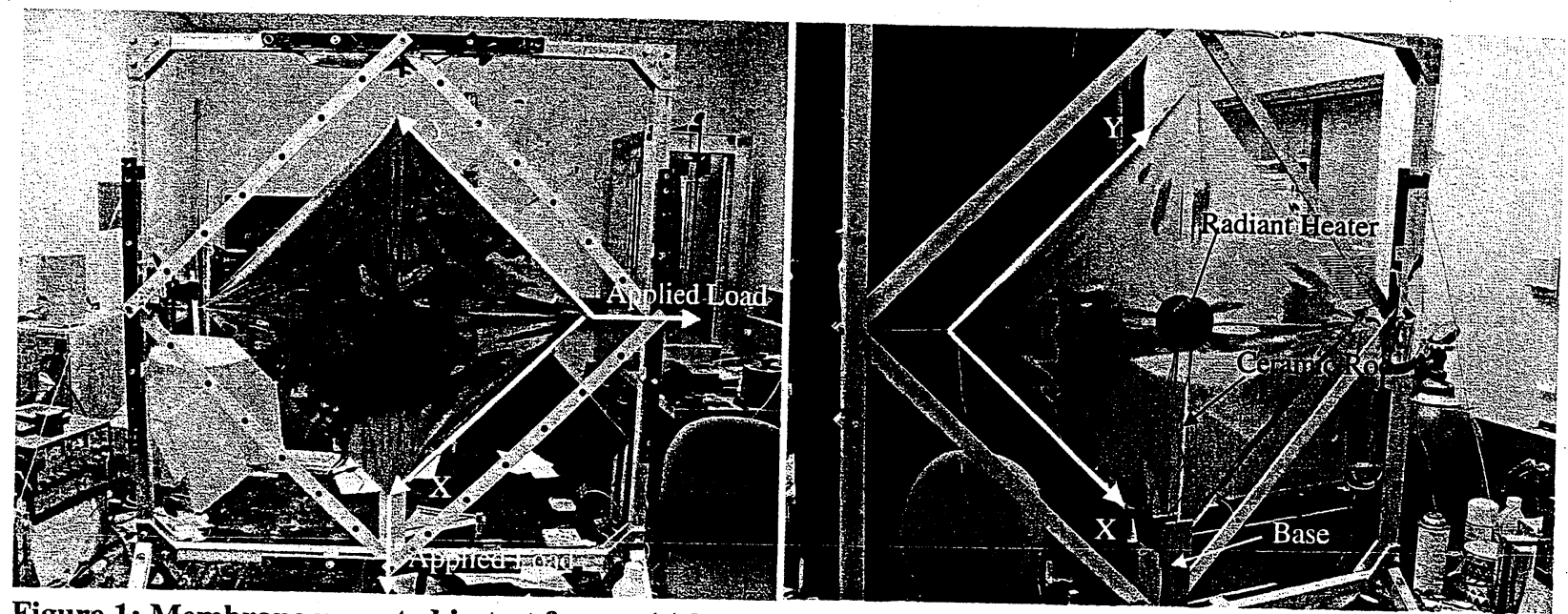

Figure 1: Membrane mounted in test frame: (a) back-side of membrane is shown and (b) front side of membrane with heater.
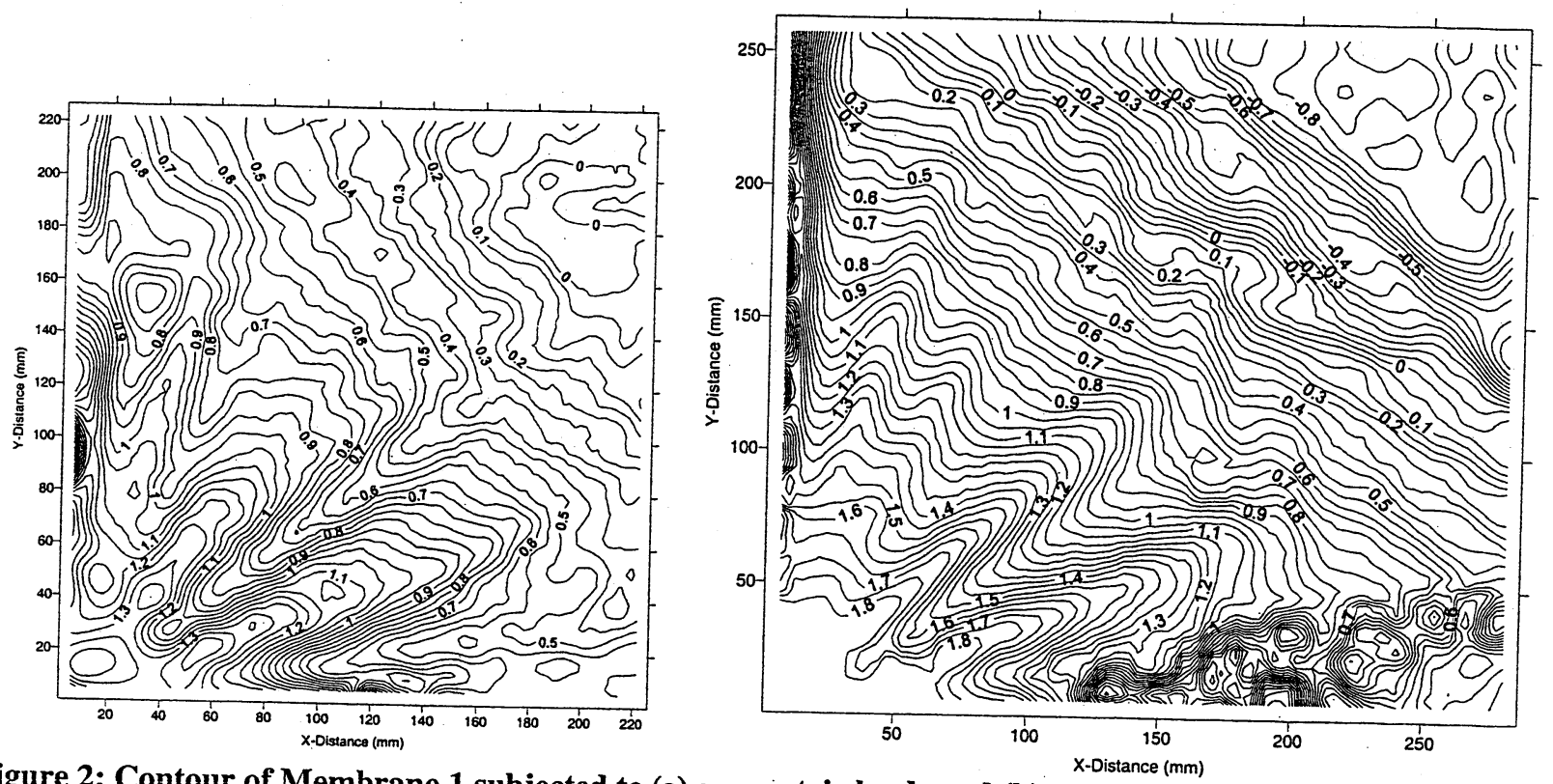

Figure 2: Contour of Membrane 1 subjected to (a) symmetric loads and (b) asymmetric loads. Note that the plots have been scaled such that the $x$ and $y$ axes are similar.
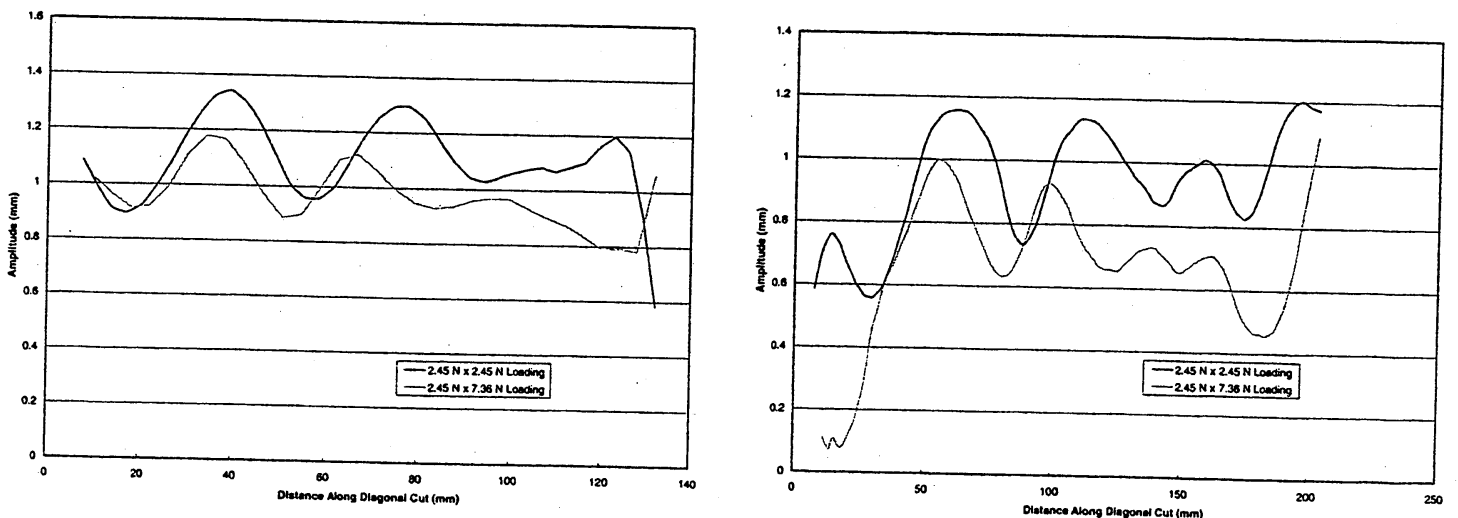

Figure 3: Amplitude data taken from cross sections of the wrinkled membrane: (a) data along a diagonal line from $X=100 \mathrm{~mm}$ to $Y=100 \mathrm{~mm}$ and (b) data along a diagonal line from $X=150 \mathrm{~mm}$ to $Y=150 \mathrm{~mm}$. 

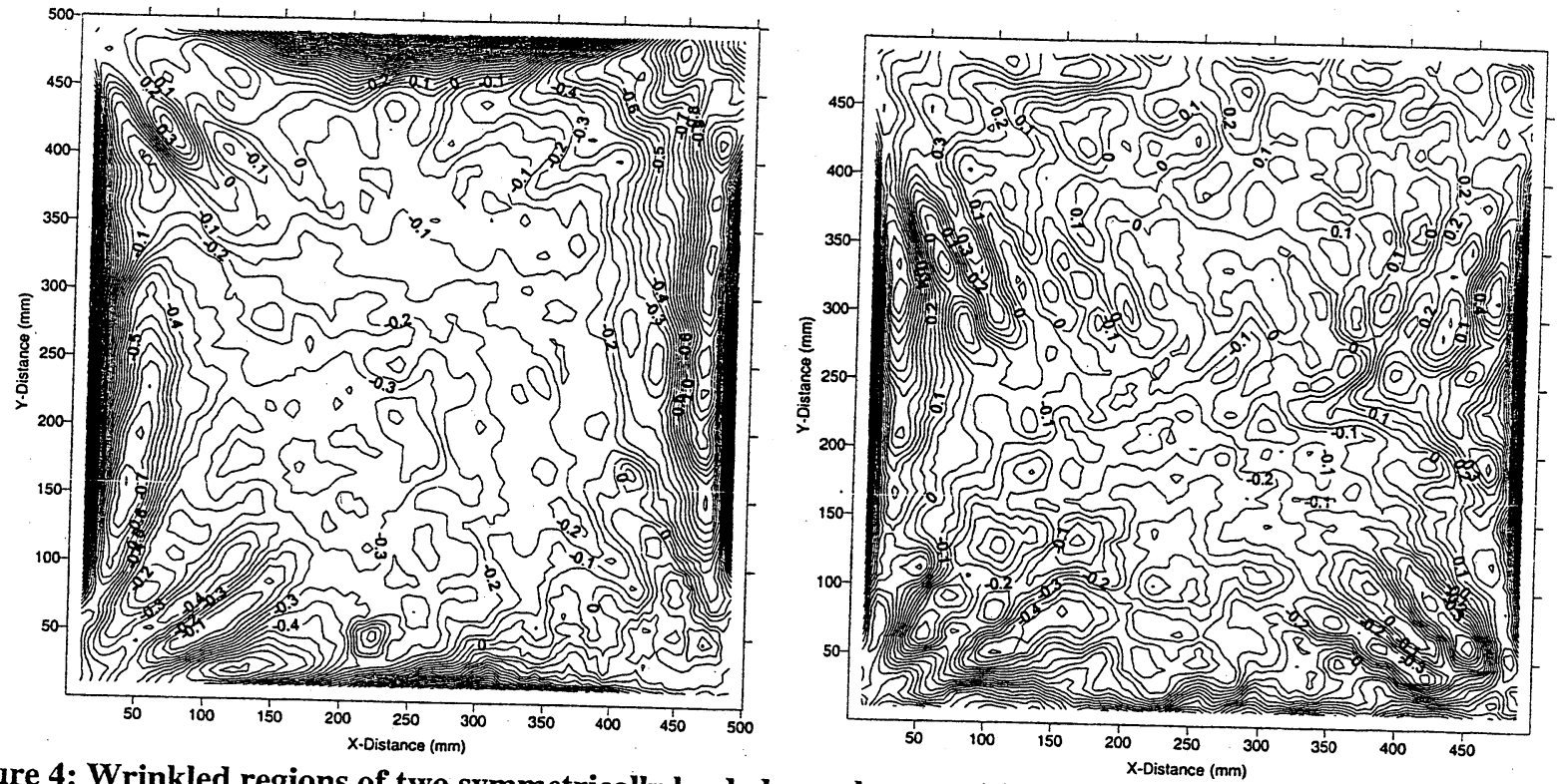

Figure 4: Wrinkled regions of two symmetrically loaded membranes: (a) membrane 1 symmetrically loaded with $2.45 \mathrm{~N}$ forces and (b) membrane 2 symmetrically loaded with $2.45 \mathrm{~N}$ forces.
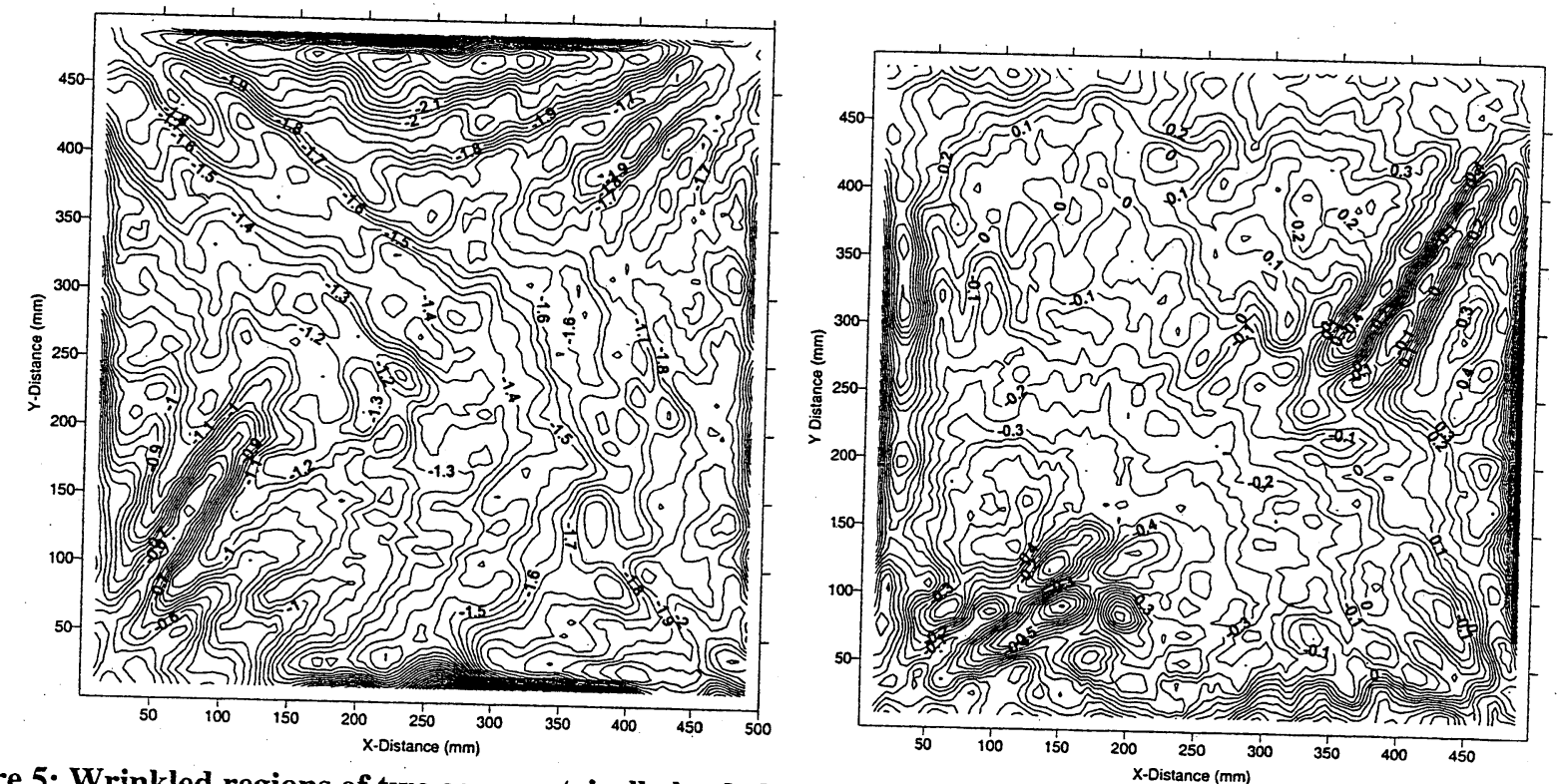
Figure 5: Wrinkled regions of two asymmetrically loaded membranes: (a) membrane 1 asymmetrically
loaded with $2.45 \mathrm{~N}$ and $7.36 \mathrm{~N}$ Forces and (b) membrane 2 asymmetrically loaded with $2.45 \mathrm{~N}$ and $7.36 \mathrm{~N}$
Forces. 


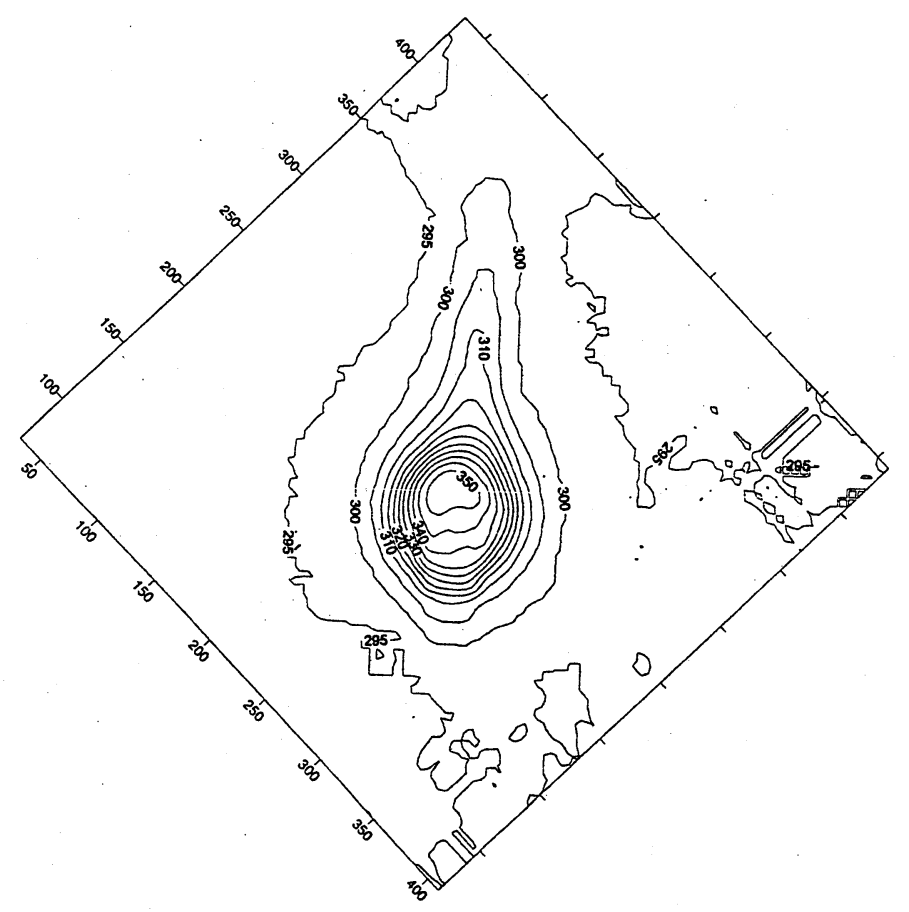

Figure 6: Measured temperature distribution near the center of the heated membrane.
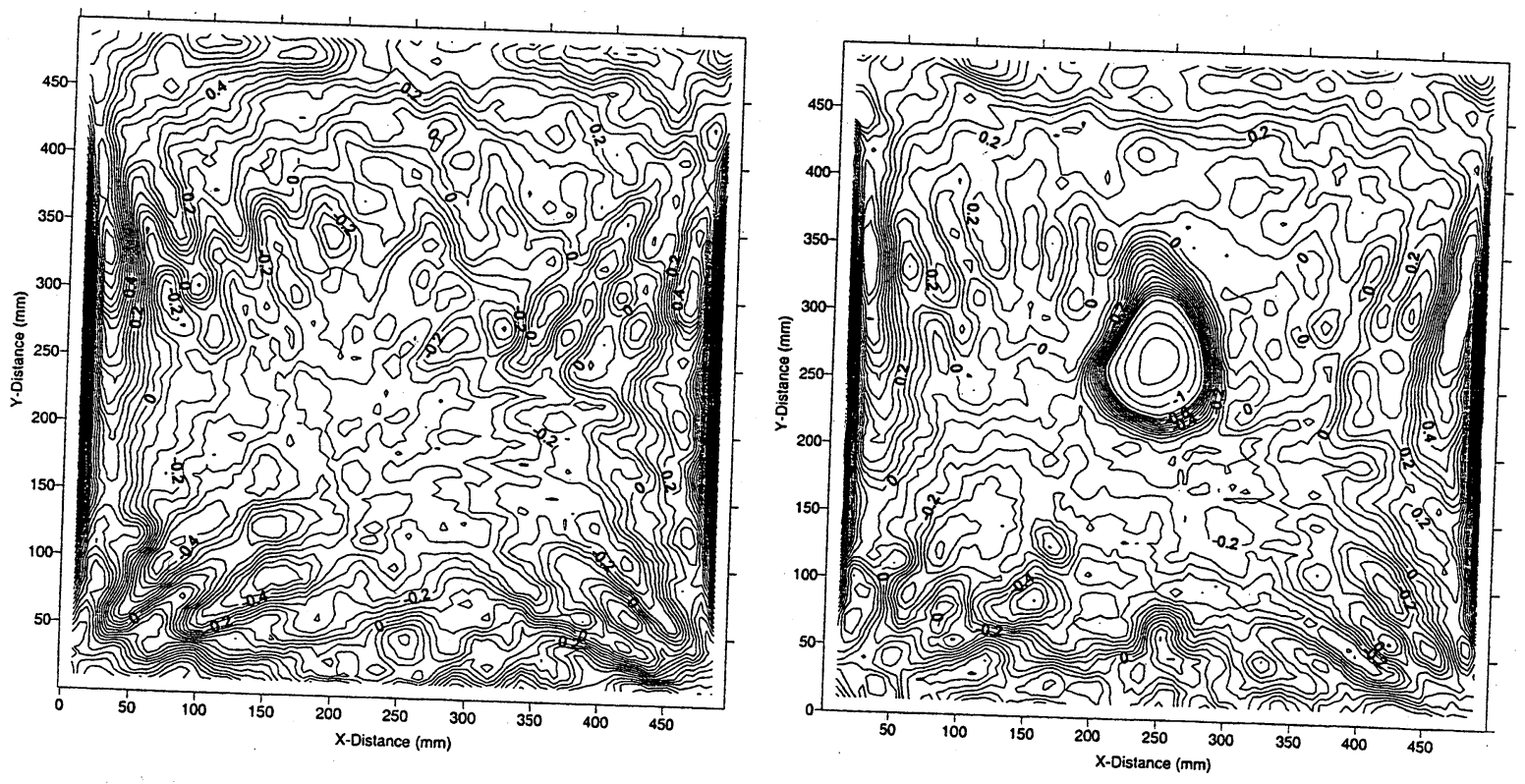

Figure 7: Wrinkle regions for symmetrically loaded unheated and heated membranes: (a) symmetrically
loaded membrane prior to heating and (b) symmetrically loaded loaded membrane prior to heating and (b) symmetrically loaded membrane subjected to heating in center. 

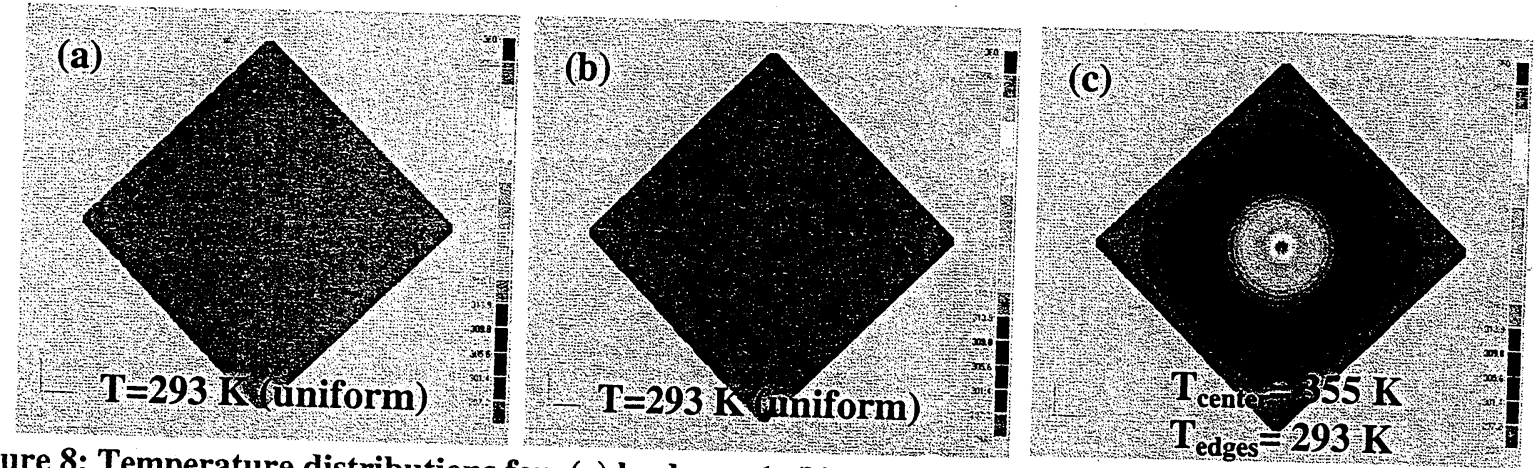

Figure 8: Temperature distributions for: (a) load case 1, (b) load case 2, and (c) load case 3.
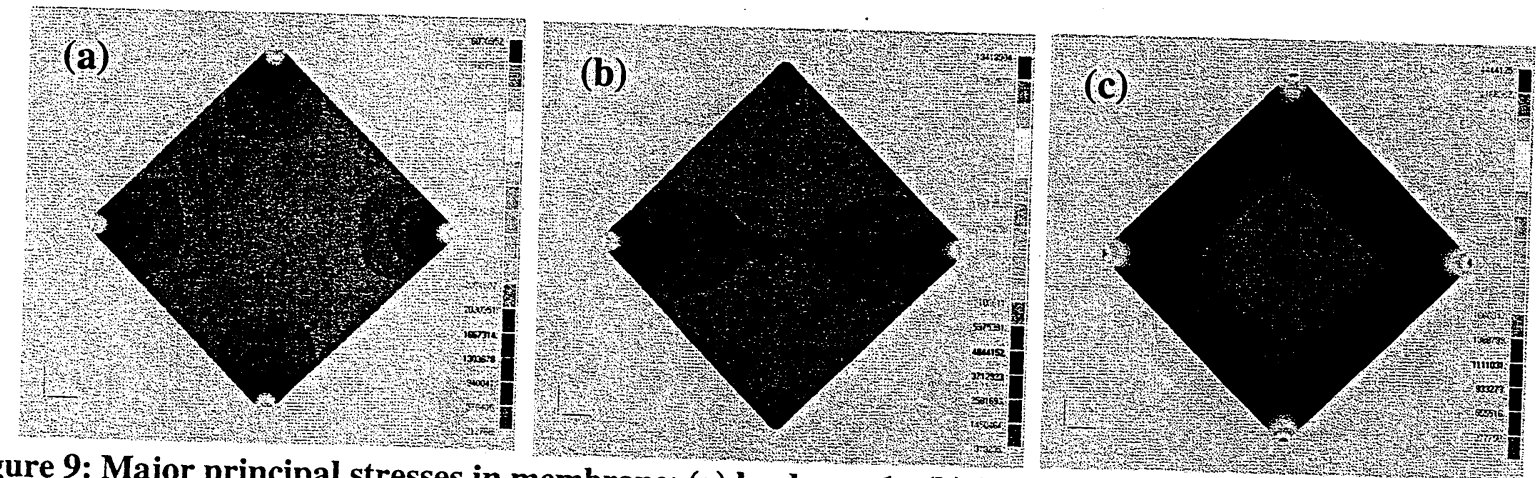

Figure 9: Major principal stresses in membrane: (a) load case 1, (b) load case 2, and (c) load case 3.
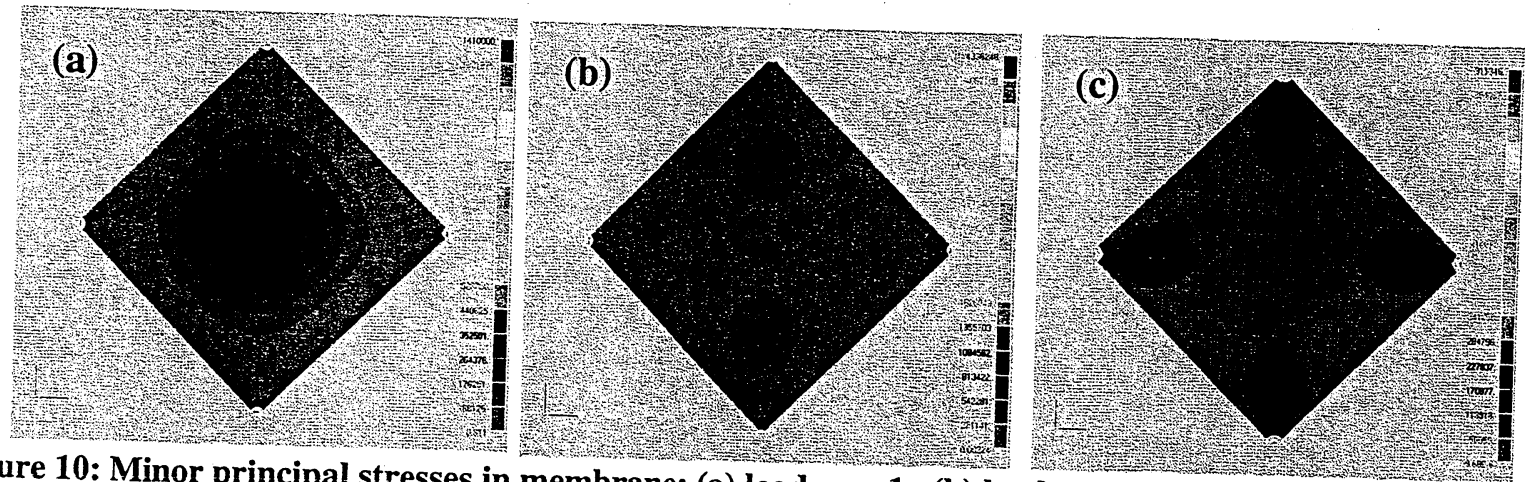

Figure 10: Minor principal stresses in membrane: (a) load case 1, (b) load case 2, and (c) load case 3 .
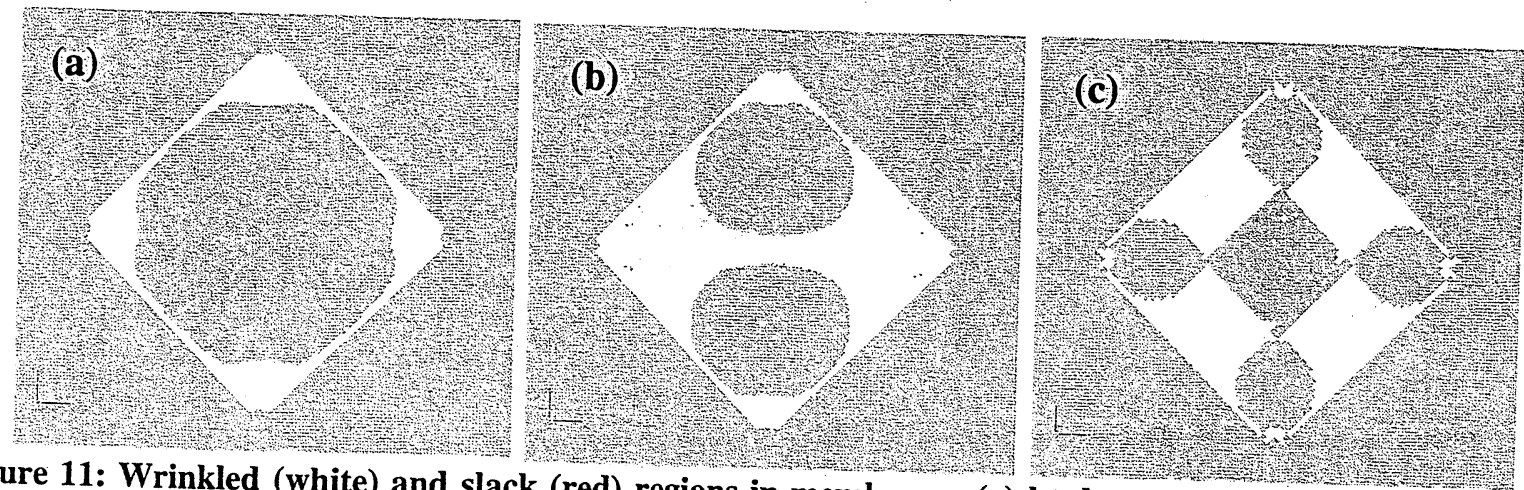

Figure 11: Wrinkled (white) and slack (red) regions in membrane: (a) load case 1 , (b) load case 2 , and (c)
load case 3. 


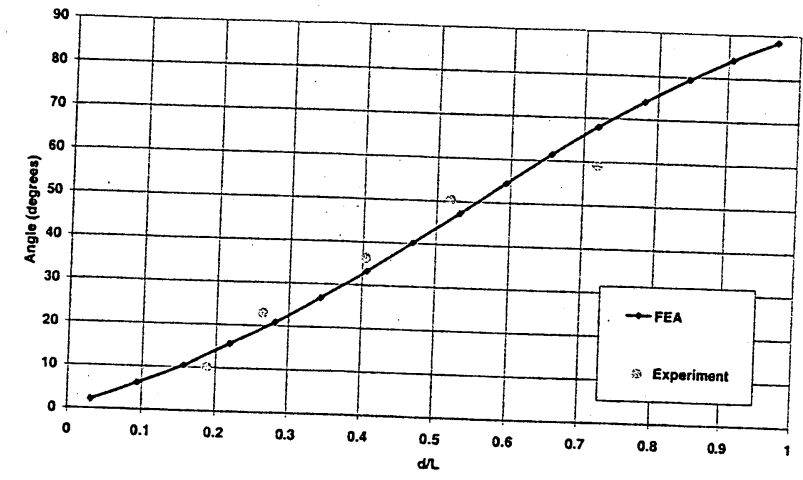

Figure 12: Measured and predicted wrinkle angles: asymmetrically loaded membrane.

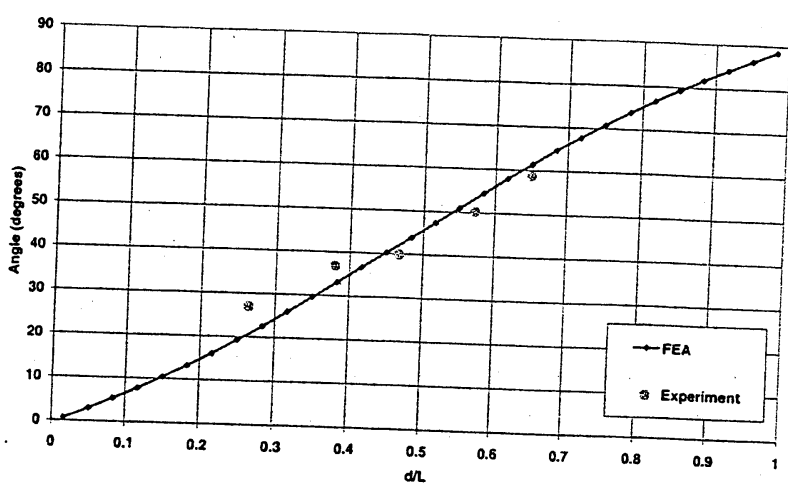

(a) symmetrically loaded membrane and (b)

Table 1: Load Cases for asymmetric, isothermal mechanical loading.

\begin{tabular}{|c|c|c|c|c|}
\hline \multirow{2}{*}{ Load Case } & \multicolumn{2}{|c|}{ Side Load (N) } & \multicolumn{2}{c|}{ Bottom Load (N) } \\
\cline { 2 - 5 } & Desired & Actual & Desired & Actual \\
\hline 1 & 2.45 & 2.45 & 2.45 & 2.45 \\
\hline 2 & 7.36 & 7.34 & 2.45 & 2.45 \\
\hline 3 & 2.45 & 2.45 & 2.45 & 2.45 \\
\hline
\end{tabular}

Table 2: Wrinkle angles for peaks and valleys near left corner

\begin{tabular}{|c|c|c|}
\hline Load Case & \begin{tabular}{|l|} 
Left Corner \\
Peak Angles \\
\end{tabular} & $\begin{array}{l}\text { Left Corner } \\
\text { Valley Angles }\end{array}$ \\
\hline \multirow[t]{3}{*}{$2.45 \mathrm{~N} \times 2.45 \mathrm{~N}$} & $22.89^{\circ}$ & $9.99^{\circ}$ \\
\hline & $50.37^{\circ}$ & $36.24^{\circ}$ \\
\hline & & $58.97^{\circ}$ \\
\hline \multirow[t]{3}{*}{$2.45 \mathrm{~N} \times 7.36 \mathrm{~N}$} & $26.91^{\circ}$ & $36.84^{\circ}$ \\
\hline & $39.81^{\circ}$ & $50.14^{\circ}$ \\
\hline & $58.67^{\circ}$ & \\
\hline
\end{tabular}

Table 3: Summary of predicted membrane stresses.

\begin{tabular}{|l|c|c|c|c|}
\hline Stress Results & Units & Load Case 1 & Load Case 2 & Load Case 3 \\
\hline$\sigma x$ (center) & $\mathrm{MPa}$ & 0.2 & 1.0 & 0.0 \\
\hline$\sigma y$ (center) & $\mathrm{MPa}$ & 0.2 & 0.0 & 0.0 \\
\hline$\sigma_{\text {major }}(\max )$ & $\mathrm{MPa}$ & 4.5 & 13.2 & 4.4 \\
\hline$\sigma_{\text {major }}(\min )$ & $\mathrm{MPa}$ & 0.2 & 0.3 & 0.0 \\
\hline$\sigma_{\text {minor }}(\max )$ & $\mathrm{MPa}$ & 1.1 & 3.2 & 0.9 \\
\hline$\sigma_{\text {minor }}(\min )$ & $\mathrm{MPa}$ & 0.0 & 0.0 & 0.0 \\
\hline
\end{tabular}


